\title{
Giant fluctuations of local magnetoresistance of organic spin valves
}

\author{
R. C. Roundy ${ }^{1 *}$, D. Nemirovsky ${ }^{2}$, V. Kagalovsky ${ }^{2}$, and M. E. Raikh ${ }^{1}$ \\ ${ }^{1}$ Department of Physics and Astronomy, University of Utah, Salt Lake City, UT 84112, USA \\ ${ }^{2}$ Sami Shamoon College of Engineering, Beer-Sheva, 84100 Israel \\ *email: rroundy@physics.utah.edu, phone: (801) 585-5017
}

\begin{abstract}
We generalize the seminal Julliere formula for the tunnel magnetoresistance (TMR) of a spin valve to include the spin memory loss of an electron in course of travel between the electrodes. This generalized version applies locally and for arbitrary mechanism of the spin dephasing. On the basis of the generalized formula we demonstrate that the distribution of TMR along the surface of magnetized electrodes is very broad and includes the sign reversals.
\end{abstract}

Keywords: Spin valve; Tunnel magnetoresistance; Spin evolution matrix; Spin-flip probability; Hyperfine magnetic fields

\section{INTRODUCTION.}

The spin valve effect, which is the dependence of the resistance of an $\mathrm{F} / \mathrm{N} / \mathrm{F}$ sandwich on the mutual orientations of ferromagnetic (F) electrodes, was uncovered ${ }^{1}$ as early as 1985, long before it became appealing for "spintronics" applications. Since 2002, experiments utilizing the spin valve effect have been reported with a wide range of materials used as the device active layer $(\mathrm{N})$. These materials range from aluminum ${ }^{2}$ to gallium arsenide ${ }^{3}$ to graphene ${ }^{4}$. Organic spin valves (OSV) were first fabricated in 2004 and instantaneously became the object of extensive studies $^{5-13}$. In OSV the the role of active layer is played by an organic semiconductor. The physics of transport in the two types of spin valves is distinctively different. In conventional spin valves the carriers travel by diffusion. In OSV the mobility of the carriers is extremely low, and the transport is due to hops from site to site. This low mobility is compensated by a very long spin relaxation time in organic semiconductors. This is because the spin-orbit coupling in carbon-based materials is very weak. Thus, despite a long travel time a carrier arriving to the bottom electrode, Fig. 1, maintains the memory about its initial spin direction.

The physics of the spin memory loss in OSV is also distinctively different from conventional spin valves. Instead of flipping spin in course of scattering from disorder ${ }^{14}$, a carrier spin undergoes precessions around local fields while sitting on a site and waiting for the subsequent hop $^{15}$. These local (hyperfine) fields are created by the nuclei surrounding the site. With the hyperfine fields being random, visiting these sites leads to the gradual loss of the spin memory. $9,16-19$

In realistic OSV the processes of the spin-memory loss are more complex and can be conveniently divided into two groups:

(i) interfacial processes, which take place at the interfaces between the electrodes and active layer ${ }^{20-26}$, and

(ii) intralayer processes, which exist even if the interfaces are ideal. ${ }^{16-18}$ These processes caused by hyperfine fields were experimentally proven ${ }^{9}$ to dominate the spinmemory loss in the bulk.

On the theoretical side, the effectiveness of the OSV

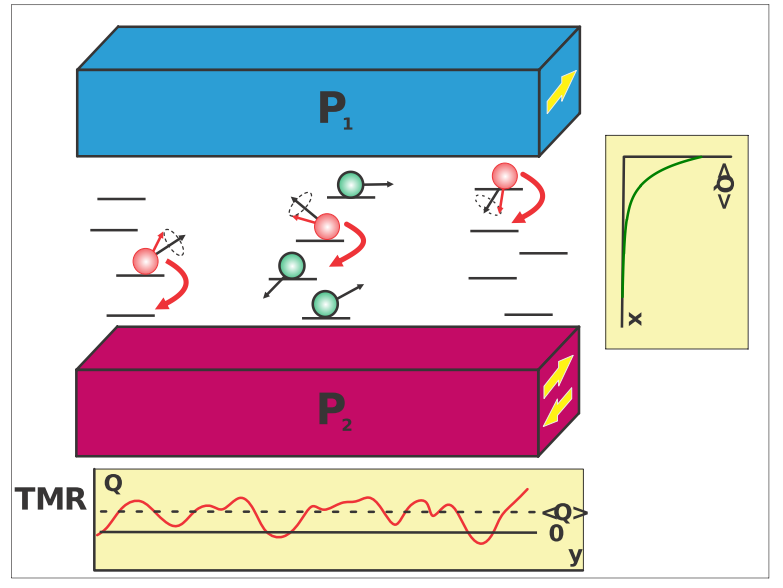

FIG. 1: (Color online) Schematic illustration of an OSV with a thin active layer, so that the transport is along independent chains. Electrode polarizations, $P_{1,2}$, are indicated in yellow. The in-plane components of hyperfine fields are depicted with black arrows. Below: A cartoon of local TMR along the $y$ direction; the classical value is indicated with a dashed line. Right: Decay of the average polarization across the active layer is shown.

performance is quantified by tunnel magnetoresistance (TMR) given by a so-called modified Julliere's formula ${ }^{27}$, see e.g. the review Ref. 28,

$$
\mathrm{TMR}=\frac{2 P_{1} P_{2} \exp \left(-d / \lambda_{s}\right)}{1-P_{1} P_{2} \exp \left(-d / \lambda_{s}\right)},
$$

where $P_{1}, P_{2}$ stand for polarizations of the electrodes. The difference from the original Julliere's formula ${ }^{27}$ is the exponential factor $Q=\exp \left(-d / \lambda_{s}\right)$ describing the spinmemory loss over the active layer of thickness, $d$. Processes (i) can be incorporated into Eq. (1) by appropriately modifying $P_{1}, P_{2}$. Processes (ii), on the other hand, are reflected in Eq. (1) via the factor $Q=\exp \left(-d / \lambda_{s}\right)$, where $\lambda_{s}$ is the spin diffusion length. The meaning of $Q$ is the polarization of electrons at $x=d$, provided that at $x=0$ they are fully polarized. Encoding the processes (ii) into $Q=\exp \left(-d / \lambda_{s}\right)$ implies that the spin polarization of electrons falls off homogeneously and monotonically with coordinate $x$, see Fig. 1 . 
The prime message of the present paper is that the factor $Q$, captures the spin memory loss only on average. The local value of $Q$ fluctuates strongly from point to point and takes values in the domain $-1<Q<1$. On the physical level, the local value of TMR in the absence of interfacial effects, is the fingerprint of hyperfine-field configuration along a given current path. The origin of strong local fluctuations of TMR is quantum-mechanical interference of amplitudes ${ }^{29}$ of subsequent spin rotations accompanying the inelastic hops of the electron which has been routinely neglected in earlier studies.

\section{REFINEMENT OF JULLIERE'S FORMULA}

In order to study the fluctuations of the local TMR the expression (1) should be generalized to capture a given path of electron between the electrodes, i.e. to an arbitrary configuration of the hyperfine fields encountered by the electron along the path. Below we demonstrate that the corresponding generalization reads

$$
\mathrm{TMR}=\frac{2 \mathcal{P}_{1} \mathcal{P}_{2}\left(1-2 P_{\mathrm{sf}}\right)}{1-\mathcal{P}_{1} \mathcal{P}_{2}\left(1-2 P_{\mathrm{sf}}\right)}
$$

where $P_{\mathrm{sf}}$ is the probability that electron flips its spin over the distance $d$. Then Eq. (2) applies even when the spin rotation in course of a scattering event is not small, i.e. the initial spin orientation is "forgotten" after only a few events.

The derivation of Eq. (2) goes as follows. For spinindependent unidirectional transport the current between the electrodes can be viewed as a sequence of cycles

$$
\begin{aligned}
I(T)=\delta\left(T-T_{1}\right)+ & \delta\left(T-T_{1}-T_{2}\right) \\
& +\delta\left(T-T_{1}-T_{2}-T_{3}\right)+\cdots,
\end{aligned}
$$

where $T_{i}$ is a random waiting time for the next electron to be transferred between $L$ and $R$. Suppose now that the left electrode is polarized $\uparrow$, while the right electrode is polarized $\downarrow$. Then the electron transfer requires a spinflip, and Eq. (3) should be modified as

$$
\begin{array}{r}
I_{\uparrow \downarrow}(T)=P_{\mathrm{sf}}\left(T_{1}\right) \delta\left(T-T_{1}\right)+P_{\mathrm{sf}}\left(T_{2}\right) \delta\left(T-T_{1}-T_{2}\right) \\
+P_{\mathrm{sf}}\left(T_{3}\right) \delta\left(T-T_{1}-T_{2}-T_{3}\right)+\cdots,
\end{array}
$$

where $P_{\mathrm{sf}}(T)$ is a quantum-mechanical probability that after a composite process with duration $T$ the electron flips its spin. For the situation considered in this paper the composite process is an inelastic two-hop tunneling. To calculate the average current one should take the limit of large $T$ and average over the compound waiting times, $T_{i}$, with distribution function $F(T)$. This averaging is convenient to perform ${ }^{30}$ using the integral representation of the $\delta$-function. Then the sum Eq. (4) turns into a geometrical progression, the summation of which yields

$$
\left\langle I_{\uparrow \downarrow}(T)\right\rangle=\int \frac{d \alpha}{2 \pi} e^{i \alpha T} \frac{\left\langle P_{\mathrm{sf}}\left(T^{\prime}\right) \exp \left(-i \alpha T^{\prime}\right)\right\rangle}{1-\left\langle\exp \left(-i \alpha T^{\prime}\right)\right\rangle} .
$$

In the limit $T \rightarrow \infty$ one can set $\alpha=0$ in the numerator and expand the denominator to the lowest order. After that the integration over $\alpha$ can be easily performed leading to the natural result

$$
\left\langle I_{\uparrow \downarrow}\right\rangle=\frac{\left\langle P_{\mathrm{sf}}\right\rangle}{\langle T\rangle},
$$

where $\left\langle P_{\text {sf }}\right\rangle$ is defined as

$$
\left\langle P_{\mathrm{sf}}\right\rangle=\int P_{\mathrm{sf}}(T) F(T) d T .
$$

For a particular case of a two-hop transport we have $T=$ $t_{1}+t_{2}$, where $t_{1}$ and $t_{2}$ are distributed with $f_{1,2}(t)=$ $\frac{1}{\tau_{1,2}} \exp \left(-t / \tau_{1,2}\right)$. Then Eq. (7) assumes the form

$$
\left\langle P_{\mathrm{sf}}\right\rangle=\int_{0}^{\infty} d t_{1} \int_{0}^{\infty} d t_{2} P_{\mathrm{sf}}\left(t_{1}, t_{2}\right) f_{1}\left(t_{1}\right) f_{2}\left(t_{2}\right) .
$$

This is exactly the quantity calculated in Sect. II. From Eq. (6) we conclude that for calculation of average current one should multiply this quantity by $1 /\langle T\rangle$, which is the current between unpolarized electrodes.

From the same reasoning we confirm that opposite directions of polarization of the electrodes the current is equal to $I_{\uparrow \uparrow}=\left(1-\left\langle P_{\mathrm{sf}}\right\rangle\right) /\langle T\rangle$. Therefore the expression for TMR with completely polarized electrodes takes the form

$$
\mathrm{TMR}=\frac{I_{\uparrow \uparrow}-I_{\uparrow \downarrow}}{I_{\uparrow \downarrow}}=\frac{1}{P_{\mathrm{sf}}}-2 .
$$

For partial polarization of electrodes with concentrations $\mathcal{N}_{\uparrow}, \mathcal{N}_{\downarrow}$ of $\uparrow$ and $\downarrow$ electrons in the left electrode and $n_{\uparrow}, n_{\uparrow}$ in the right electrode, the general expressions for $I_{\uparrow \uparrow}$ and $I_{\uparrow \downarrow}$ can be presented as

$$
\begin{aligned}
& I_{\uparrow \uparrow}=\Gamma_{\uparrow \uparrow}\left(\mathcal{N}_{\uparrow} n_{\uparrow}+\mathcal{N}_{\downarrow} n_{\downarrow}\right)+\Gamma_{\uparrow \downarrow}\left(\mathcal{N}_{\uparrow} n_{\downarrow}+\mathcal{N}_{\downarrow} n_{\uparrow}\right), \\
& I_{\uparrow \downarrow}=\Gamma_{\uparrow \uparrow}\left(\mathcal{N}_{\uparrow} n_{\downarrow}+\mathcal{N}_{\downarrow} n_{\uparrow}\right)+\Gamma_{\uparrow \downarrow}\left(\mathcal{N}_{\uparrow} n_{\uparrow}+\mathcal{N}_{\downarrow} n_{\downarrow}\right),
\end{aligned}
$$

where $\Gamma_{\uparrow \uparrow}$ and $\Gamma_{\uparrow \downarrow}$ are the rates for the transfer processes from $\uparrow$ to $\uparrow$ and from $\uparrow$ to $\downarrow$. These rates are the characteristics of the active layer and do not depend on the polarizations of the electrodes. Naturally, we have $\Gamma_{\uparrow \uparrow}=\Gamma_{\downarrow \downarrow}$ and $\Gamma_{\uparrow \downarrow}=\Gamma_{\downarrow \uparrow}$. The expression Eq. (2) follows from Eqs. (10) and (11) in two steps. We relate the concentration via the degrees of polarization as

$$
\mathcal{P}_{1}=\frac{\mathcal{N}_{\uparrow}-\mathcal{N}_{\downarrow}}{\mathcal{N}_{\uparrow}+\mathcal{N}_{\downarrow}}, \quad \mathcal{P}_{2}=\frac{n_{\uparrow}-n_{\downarrow}}{n_{\uparrow}+n_{\downarrow}},
$$

yielding

$$
\mathrm{TMR}=\frac{2 \mathcal{P}_{1} \mathcal{P}_{2}\left(\Gamma_{\uparrow \uparrow}-\Gamma_{\uparrow \downarrow}\right)}{\left(\Gamma_{\uparrow \uparrow}+\Gamma_{\uparrow \downarrow}\right)-\mathcal{P}_{1} \mathcal{P}_{2}\left(\Gamma_{\uparrow \uparrow}-\Gamma_{\uparrow \downarrow}\right)} .
$$

Finally, we relate the rates $\Gamma_{\uparrow \uparrow}, \Gamma_{\uparrow \downarrow}$ via $P_{\text {sf }}$ as

$$
\frac{\Gamma_{\uparrow \uparrow}-\Gamma_{\uparrow \downarrow}}{\Gamma_{\uparrow \uparrow}+\Gamma_{\uparrow \downarrow}}=1-2 P_{\mathrm{sf}},
$$

and arrive to Eq. (2). 


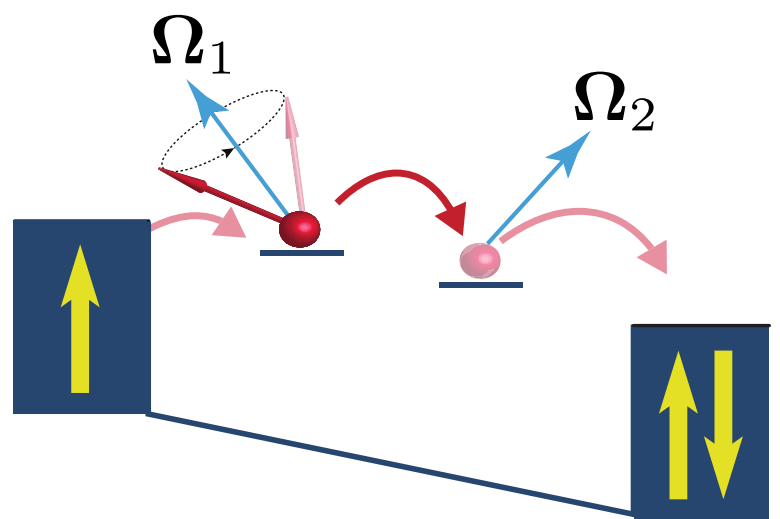

FIG. 2: (Color online) Illustration of the regime of transport between ferromagnetic electrodes $\mathrm{L}$ and $\mathrm{R}$, dominated by hops via intermediate sites 1 and 2 . Spin precession in the hyperfine fields takes place while electron waits for the hops $1 \rightarrow 2$ and $2 \rightarrow \mathrm{R}$. Bias is assumed large, so that all hops are unidirectional.

\section{DISCUSSION}

To illustrate nontrivial consequences of the generalized TMR Eq. (2) assume that electron makes only two stops on the way between the electrodes and is thus exposed to two different hyperfine fields, $\boldsymbol{\Omega}_{1}$ and $\boldsymbol{\Omega}_{2}$, see Fig. 2 .

The evolution of spin is described by the product of the unitary matrices $U\left(t_{2}\right) U\left(t_{1}\right)$, where the matrix $U(t)$ is defined as

$$
U(t)=\left[\begin{array}{cc}
\cos \alpha-i \frac{\Omega_{z}}{\Omega} \sin \alpha & -i \frac{\Omega_{-}}{\Omega} \sin \alpha \\
-i \frac{\Omega_{+}}{\Omega} \sin \alpha & \cos \alpha+i \frac{\Omega_{z}}{\Omega} \sin \alpha
\end{array}\right], \alpha=\frac{\Omega t}{2},
$$

where $t_{1}$ and $t_{2}$ are the times spent in $\boldsymbol{\Omega}_{1}$ and $\boldsymbol{\Omega}_{2}$, respectively; $\Omega_{ \pm}=\Omega_{x} \pm i \Omega_{y}$. The spin-flip amplitude is given by a non-diagonal element, $A_{\uparrow \downarrow}=-i \frac{\Omega_{+}}{\Omega} \sin \left(\frac{\Omega t}{2}\right)$. In calculation of $P_{\mathrm{sf}}$ the Poissonian averaging over the waiting times should be performed ${ }^{29}$. The result can be conveniently presented as a sum of two contributions, the incoherent contribution, $P_{\text {incoh }}$, and the interference contribution, $P_{\text {int }}$, i.e.

$$
P_{\text {sf }}=P_{\text {incoh }}+P_{\text {int }} .
$$

The expressions for $P_{\text {incoh }}$ and $P_{\text {int }}$ are

$$
\begin{gathered}
P_{\mathrm{incoh}}=p_{\mathrm{sf}}^{(1)}\left(1-p_{\mathrm{sf}}^{(2)}\right)+\left(1-p_{\mathrm{sf}}^{(1)}\right) p_{\mathrm{sf}}^{(2)}, \\
P_{\mathrm{int}}=\sqrt{p_{\mathrm{sf}}^{(1)}\left(1-2 p_{\mathrm{sf}}^{(1)}\right) p_{\mathrm{sf}}^{(2)}\left(1-2 p_{\mathrm{sf}}^{(2)}\right)} \cos \phi,
\end{gathered}
$$

where $p_{\mathrm{sf}}^{(1)}$ are $p_{\mathrm{sf}}^{(2)}$ are the partial spin-flip probabilities in $\boldsymbol{\Omega}_{1}$ and $\boldsymbol{\Omega}_{2}$, respectively. The angle $\phi$ is determined by the mutual orientations between $\boldsymbol{\Omega}_{1}$ and $\boldsymbol{\Omega}_{2}$. The expression Eq. (17) has a transparent interpretation in probabilistic terms. The interference contribution comes from the combination

$$
P_{\mathrm{int}}=2\left\langle\operatorname{Re}\left(A_{\uparrow \downarrow}^{(1)} A_{\downarrow \downarrow}^{(2)} A_{\uparrow \uparrow}^{(1) *} A_{\uparrow \downarrow}^{(2) *}\right)\right\rangle_{t_{1}, t_{2}} .
$$

Consider now a particular realization: $p_{\mathrm{sf}}^{(1)}=p_{\mathrm{sf}}^{(2)}=p$, and $\phi=0$. Then we get

$$
P_{\mathrm{sf}}=2 p(1-p)+p(1-2 p)=3 p-4 p^{2} .
$$

Note that for $p=3 / 8$ the value of $P_{\text {sf }}$ is equal to $9 / 16$, i.e. it is bigger than $1 / 2$. This, in turn, suggests that for a chosen configuration of the hyperfine fields the TMR, defined by Eq. (2) is negative.

Then a natural question arises: what is the statistical weight of the hyperfine-field configurations for which the TMR is negative? The analysis in Ref. 29 shows that, for arbitrary mutual orientation of $\boldsymbol{\Omega}_{1}$ and $\boldsymbol{\Omega}_{2}$, for TMR to be negative, the partial probabilities should be sufficiently close to each other. An even more intriguing question is what is portion of the negative TMR configurations when the partial spin-flip probabilities are small, but the number of stops which electron makes on the way between the electrodes is large. It appears ${ }^{31}$ that one can find the distribution of the parameter $Q$ analytically. The message of the calculation in Ref. 31 is that, as the number of intermediate hyperfine fields encountered by electron on the way between the electrodes increases, the distribution of $P_{\mathrm{sf}}$ becomes progressively broad. In particular, a complete spin-memory loss for which the classical distribution of $Q$ is simply $\delta(Q)$, with interference taken into account, transform into the flat distribution, suggesting that all values of the local TMR are equally probable.

\section{CONCLUSION}

The factor $\exp \left(-d / \lambda_{s}\right)$ in Eq. (1) describes the spinmemory loss only on average. In the present paper we predict that local measurements of TMR would reveal a sizable portion of the contact area in which TMR is negative. The microscopic expression for the spin-flip probability with we use throughout the paper reads ${ }^{14}$

$$
p_{\mathrm{sf}}=\frac{1}{2} \frac{\left(\Omega^{2}-\Omega_{z}^{2}\right) \tau^{2}}{1+\Omega^{2} \tau^{2}},
$$

where $\tau$ is the transit time for electron hopping. It is seen from Eq. (21) that $p_{\text {sf }}$ depends strongly on the relation between the precession frequency, $\Omega$, and the transit hopping time, $\tau$. The typical values of $\Omega$ in organic semiconductors are well defined and constitutes $\sim 10^{8}$ $\mathrm{Hz}$, which correspond to the magnitude of the hyperfine field $\sim 1 \mathrm{mT}$. On the other hand, the values of $\tau$ vary strongly from material to material. This is because $\tau$ depends exponentially on the hopping length ${ }^{15,32}$. For a specific organic semiconductor Poly[2-methoxy5-(2-ethylhexyloxy)-1,4-phenylenevinylene] (MEH-PPV) widely studied experimentally the value of $\tau$ was inferred from the echo experiment ${ }^{33}$ to be $3 \times 10^{-7} \mathrm{~s}$. 


\section{ACKNOWLEDGEMENTS}

We are grateful to Z. V. Vardeny for motivating us. Also we are extremely grateful to V. V. Mkhitaryan for reading the manuscript and very useful suggestions. This work was supported by the NSF through MRSEC DMR112125, and by the BSF Grant No. 2010030.
${ }^{1}$ M. Johnson and R. H. Silsbee, Phys. Lett. 55, 1790 (1985).

${ }^{2}$ F. J. Jedema, A. T. Filip, and B. J. van Wees, Nature (London) 410, 345 (2001).

3 S. A. Crooker, M. Furis, X. Lou, C. Adelmann, D. L. Smith, C. J. Palmstrøm, and P. A. Crowell, Science 309 2191 (2005).

${ }^{4}$ N. Tombros, C. Jozsa, M. Popinciuc, H. T. Jonkman, and B. J. van Wees, Nature (London) 448, 571 (2007).

${ }^{5}$ Z. H. Xiong, D. Wu, Z. V. Vardeny, and J. Shi, Nature (London) 427, 821 (2004).

6 S. Pramanik, C.-G. Stefanita, S. Patibandla, S. Bandyopadhyay, K. Garre, N. Harth, and M. Cahay, Nat. Nanotechnol. 2, 216 (2007).

7 V. A. Dediu, L. E. Hueso, I. Bergenti, and C. Taliani, Nat. Mater. 8, 850 (2009).

8 A. J. Drew, J. Hoppler, L. Schulz, F. L. Pratt, P. Desai, P. Shakya, T. Kreouzis, W. P. Gillin, A. Suter, N. A. Morley, V. K. Malik, A. Dubroka, K. W. Kim, H. Bouyanfif, F. Bourqui, C. Bernhard, R. Scheuermann, G. J. Nieuwenhuys, T. Prokscha, and E. Morenzoni, Nat. Mater. 8, 109 (2009).

9 T. Nguyen, G. Hukic-Markosian, F. Wang, L. Wojcik, X. Li, E. Ehrenfreund, Z. Vardeny, Nat. Mater. 9, 345 (2010).

10 T. D. Nguyen, F, Wang, X.-G. Li, E. Ehrenfreund, and Z. V. Vardeny, Phys. Rev. B 87, 075205 (2013).

11 M. Grünewald, J. Kleinlein, F. Syrowatka, F. Würthner, L.W. Molenkamp, and G. Schmidt, arXiv:1304.2911.

12 A. Riminucci, M. Prezioso, C. Pernechele, P. Graziosi, I. Bergenti, R. Cecchini, M. Calbucci, M. Solzi, V. A. Dediu, Appl. Phys. Lett. 102, 092407 (2013).

13 M. Grünewald, R. Göckeritz, N. Homonnay, F. Würthner, L. W. Molenkamp, and G. Schmidt, Phys. Rev. B 88, 085319 (2013)

14 see e.g. M. I. Dyakonov and V. I. Perel, in Optical Orientation, edited by F. Meier and B. Zakharchenya NorthHolland, Amsterdam, 1984, pp. 1171.

15 P. A. Bobbert, T. D. Nguyen, F. W. A. van Oost, B. Koopmans, and M. Wohlgenannt, Phys. Rev. Lett. 99, 216801 (2007).

16 J. J. H. M. Schoonus, P. G. E. Lumens, W. Wagemans,
J. T. Kohlhepp, P. A. Bobbert, H. J. M. Swagten, and B. Koopmans, Phys. Rev. Lett. 103, 146601 (2009).

17 P. A. Bobbert, W. Wagemans, F. A. van Oost, B. Koopmans, and M. Wohlgenannt, Phys. Rev. Lett. 102, 156604 (2009).

18 N. J. Harmon and M. E. Flatté, Phys. Rev. Lett. 110, 176602 (2013)

19 R. N. Mahato, H. Lülf, M. H. Siekman, S. P. Kersten, P. A. Bobbert, M. P. de Jong, L. De Cola, W. G. van der Wiel, Science 341, 257 (2013).

${ }^{20}$ C. Barraud, P. Seneor, R. Mattana, S. Fusil, K. Bouzehouane, C. Deranlot, P. Graziosi, L. Hueso, I. Bergenti, V. Dediu, F. Petroff, and A. Fert, Nat. Phys. 6, 615 (2010).

21 S. H. Liang, D. P. Liu, L. L. Tao, X. F. Han, and Hong Guo, Phys. Rev. B 86, 224419 (2012).

22 J. M. De Teresa, A. Barthélémy, A. Fert, J. P. Contour, R. Lyonnet, F. Montaigne, P. Seneor, and A. Vaurès, Phys. Rev. Lett. 82, 4288 (1999).

${ }^{23}$ W. Xu, G. J. Szulczewski, P. LeClair, I. Navarrete, R. Schad, G. Miao, H. Guo, and A. Gupta, Appl. Phys. Lett. 90, 072506 (2007).

${ }^{24}$ S. Mooser, J. F. K. Cooper, K. K. Banger, J. Wunderlich, and H. Sirringhaus, Phys. Rev. B 85, 235202 (2012).

25 S. Mandal and R. Pati, ACS Nano, 6, 3580 (2012).

26 M. A. Tanaka, T. Hori, K. Mibu, K. Kondou, T. Ono, S. Kasai, T. Asaka, and J. Inoue, J. Appl. Phys. 110, 073905 (2011).

27 M. Julliére, Phys. Lett. 54A, 225 (1975).

28 K. M. Alam and S. Pramanik, arXiv:1005.1118.

29 R. C. Roundy and M. E. Raikh, Phys. Rev. B, 88, 205206 (2013).

30 J. Koch, M. E. Raikh, and F. von Oppen, Phys. Rev. Lett. 95, $056801(2005)$

31 R. C. Roundy, D. Nemirovsky, V. Kagalovsky, and M. E. Raikh, Phys. Rev. Lett. 112, 226601 (2014).

32 N. J. Harmon and M. E. Flatté Phys. Rev. B 85, 075204 (2012).

33 W. J. Baker, T. L. Keevers, J. M. Lupton, D. R. McCamey, and C. Boehme, Phys. Rev. Lett. 108, 267601 (2012). 